\title{
Olecranon Apophyseal Fracture and Radial Neck Fracture in a Patient without Osteogenesis Imperfecta: A Case Report
}

Abdullah A. Alshamrani

Department of Orthopedic Surgery, King Abdulaziz Hospital, Makkah, Saudi Arabia

Corresponding Author: Abdullah A. Alshamrani, E-Mail: dr.a.alshamrani2016@gmail.com

\begin{abstract}
Background: Olecranon apophyseal fractures in children are uncommon. The bulk of these injuries are nondisplaced and therefore can be treated nonoperatively.

Objective: To study the outcome of open reduction and internal fixation of olecranon fracture.

Patients and methods: Our case report of a 9 years old girl who sustained left elbow trauma after falling directly over the elbow. Attended the outpatient clinic of the Department of Orthopedic Surgery, Alnoor Specialist Hospital, Makkah, Saudi Arabia, radiographs showed olecranon and radial neck fractures, olecranon fracture was displaced so we fix it with K-wire and tension band, while the radial neck fracture was treated conservatively.

Results: At the 12 weeks follow-up, she achieved a complete range of motion of the left elbow with good functional outcomes and with no complications. The goal in such complex fractures is to achieve a stable congruent joint with gradual rehabilitation to reach a good functional outcome.

Conclusion: open reduction and internal fixation of the olecranon fracture is the optimal treatment to achieve a complete range of motion and good functional outcome.
\end{abstract}

Keywords: Olecranon, Radial neck, Elbow injury.

\section{INTRODUCTION}

Olecranon apophyseal fractures in children are uncommon. The bulk of these injuries are nondisplaced and therefore can be treated nonoperatively.

Few published reports of children with displaced fractures of the olecranon apophysis exist, and the large majority of reports describe children with osteogenesis imperfecta. Our report is about a 9-years old, healthy, girl patient without osteogenesis imperfecta who sustained a displaced olecranon apophyseal fracture during a fall.

\section{Ethical approval:}

The patient signed informed written consent for the acceptance of the operation and publication of this case report. An approval of the study was obtained from Alnoor Specialist Hospital, Makkah, Saudi Arabia, academic and ethical committee. This work has been carried out in accordance with The Code of Ethics of the World Medical Association (Declaration of Helsinki) for studies involving humans.

\section{CASE REPORT}

A 9 years old girl not known to have any medical illnesses came to the Emergency Department complaining of left elbow pain after she falls while playing 1 hour before her presentation. The pain was severe mainly at the elbow and radiated to the mid-forearm. Associated with swelling and ecchymosis around the elbow. She could not move the elbow passively and actively due to pain. No signs of compartment syndrome.

No open wounds. The wrist and shoulder were free from injuries and have a full range of motion. Distal neurovascularity was normal. Anteroposterior, lateral, and oblique radiographs showed olecranon apophyseal fracture and radial neck fracture (Figure 1).

Then above elbow posterior splint applied. And the patient was admitted to the ward for surgical fixation. The patient underwent open reduction and internal fixation of olecranon fracture using K-wire and tension band. Using a direct posterior approach to the elbow. The ulnar nerve was dissected and protected with a vessel loop. Olecranon fracture was identified and the hematoma was evacuated and irrigation with normal saline done. Fracture reduced and fixed with two K-wire and tension band under the guidance of fluoroscopy (Figure 2,3).

After closure and dressing, a posterior splint was applied. Distal neurovascularity was intact. Radial neck fracture was treated conservatively. 


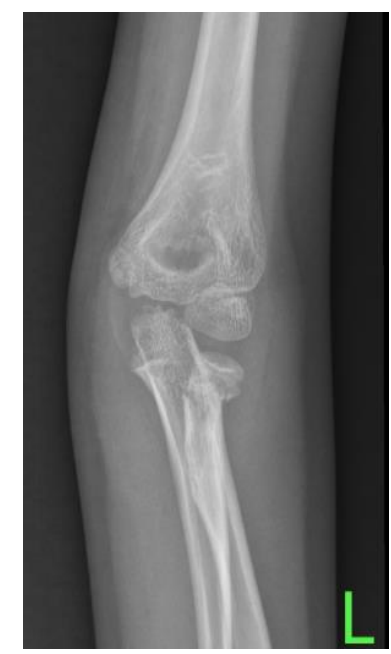

(A)

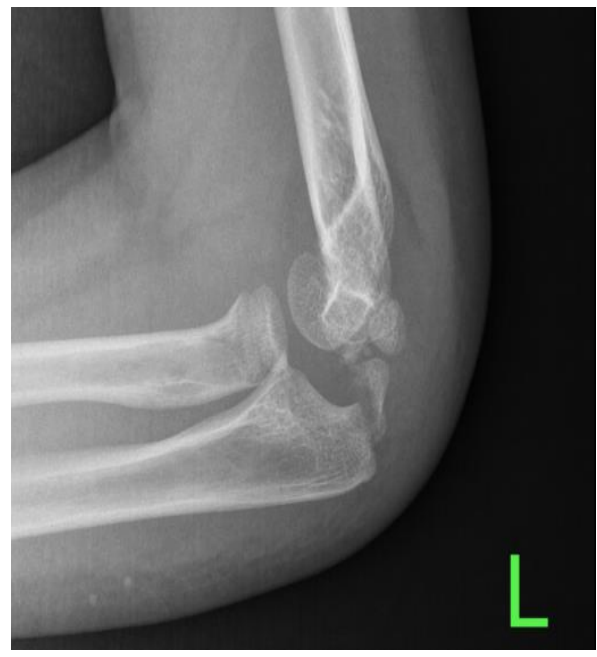

(B)

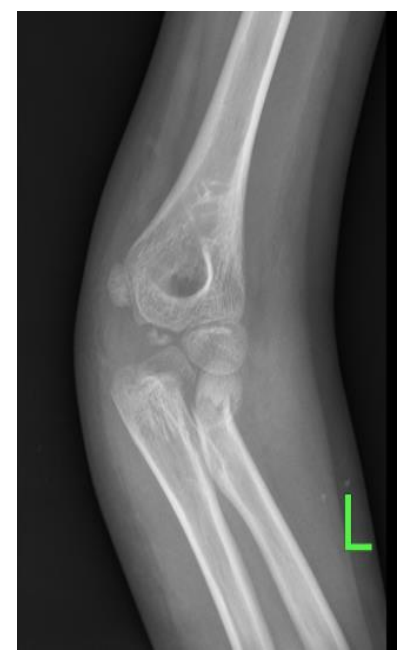

(C)

Figure 1. An anteroposterior radiograph of the left elbow showed a minimally displaced radial neck fracture (A). The lateral view demonstrates displaced transverse olecranon apophyseal fracture and also radial neck fracture is clear in this view (B). An oblique view showed more details about the geometry of both fractures.

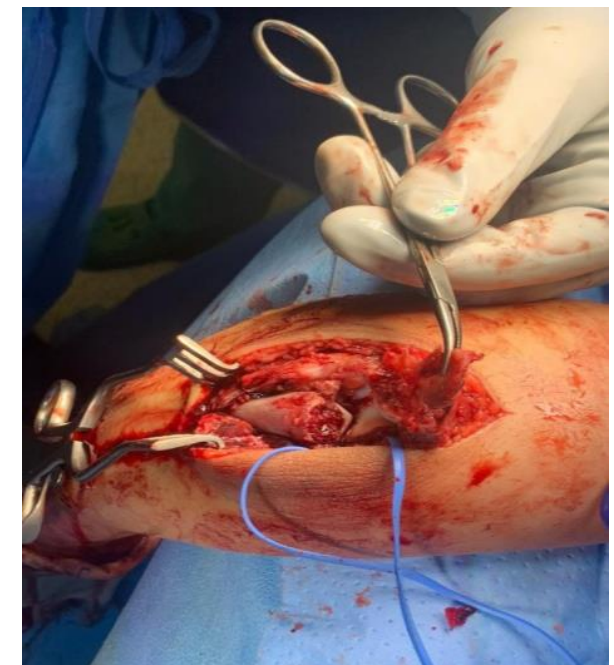

(A)

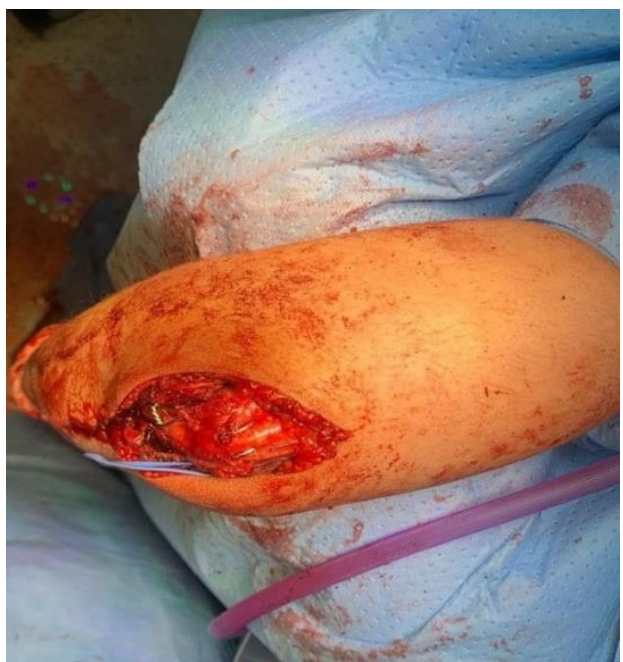

(B)

Figure 2. Intraoperative picture demonstrated the fracture fragments of the olecranon that has been cleaned and refreshed (A). After complete reduction and fixation with K-wire and tension band (B).

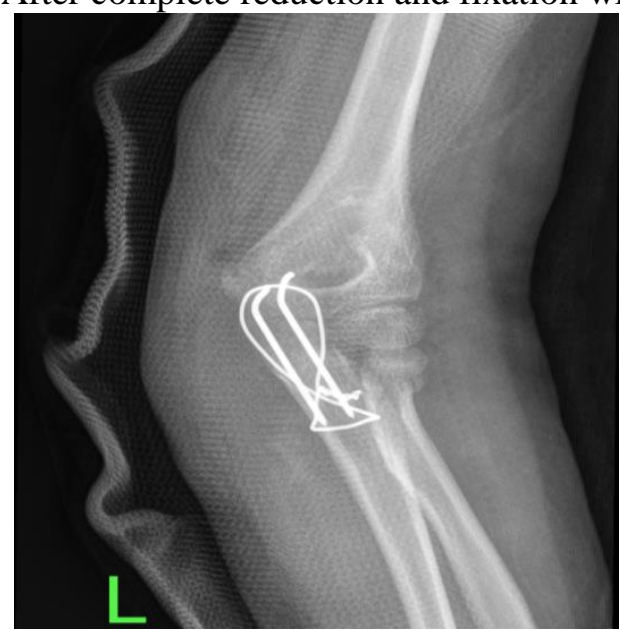

(A)

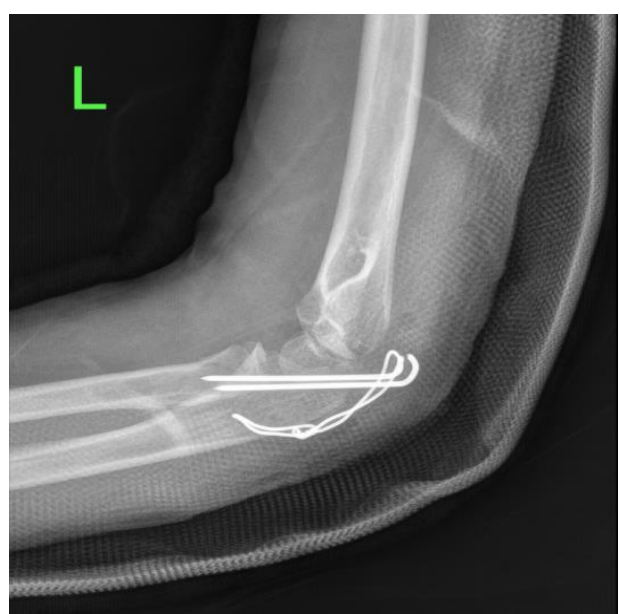

(B)

Figure 3. Immediate postoperative radiographs showed a stable fixation of olecranon using K-wire and tension band, note that the radial neck fracture treated with a reduction only, and a posterior splint was applied in 90 degrees of flexion and neutral rotation. 


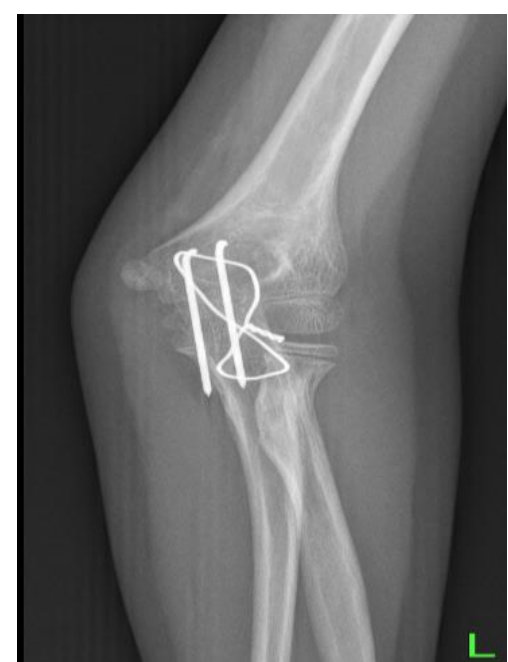

(A)

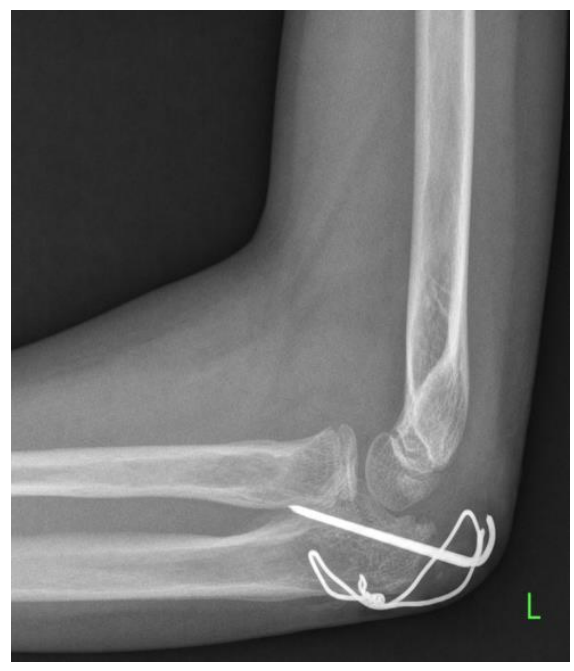

(B)

Figure 4. 12 weeks follow-up showed complete healing of olecranon fracture, and also good healing with a great remodeling of radial neck fracture with some residual angulation in the coronal plane.

\section{DISCUSSION}

The olecranon fracture is exceedingly uncommon, accounting for just around 5\% of all elbow fractures (1). The cartilaginous radial head is resistant to fracture in children, and radial neck fractures are more common in minors than head fractures ${ }^{(2)}$. Olecranon fractures are less prevalent and less severe in children than in adults due to a variety of anatomical variables. First, because the olecranon is mostly cartilage, a direct hit to the olecranon is less likely to result in a fracture, especially in younger children. Second, the olecranon's thick periosteum and thin metaphyseal cortex make it susceptible to minimally displaced greenstick fractures.

A hyperextension injury is the most common cause of olecranon fractures. However, a direct hit to the flexed elbow, hyperflexion injury, or shear force can all induce fractures ${ }^{(3)}$. Other elbow injuries are usually linked to hyperextension injuries. The associated injuries will be determined by the direction of the associated coronal plane force.

A valgus hyperextension force may produce an associated radial neck or medial epicondyle fracture.

Clinically, an olecranon fracture is usually manifested as a swollen elbow. An abrasion or contusion on the posterior aspect of the elbow may provide a clue about the nature of the injury. There may be a palpable defect posteriorly, as well as an inability to extend the elbow. When the olecranon apophysis is not ossified, the examiner may miss a fracture through the olecranon ${ }^{(4)}$. Once the diagnosis has been made, the patient and radiographs should be closely examined for associated injuries, including injuries to the radial head or neck, which have been noted to occur in a third of olecranon fractures ${ }^{(5)}$.

Nondisplaced or minimally $(\leq 3 \mathrm{~mm})$ displaced fractures can generally be managed by simple cast immobilization for 3 to 4 weeks. If the fractures are displaced (>3 mm), extraarticular, and stable, they can usually be managed by closed reduction and cast immobilization ${ }^{(6)}$.

Intraarticular fractures with more than $3 \mathrm{~mm}$ of displacement usually require open reduction and internal fixation. Generally, using a standard pin and tension band technique for displaced olecranon fractures is preferred ${ }^{(5,7)}$. Patients with osteogenesis imperfecta and displaced fractures usually require internal fixation and are prone to re-fracture after implant removal ${ }^{(8)}$. Some authors have described fixation with absorbable implants as an alternative to pins and wires. They noted fewer symptoms from hardware with this technique ${ }^{(3,9)}$.

Radial neck fractures: The greatest difficulty in treating radial neck fractures lies in determining which fractures require reduction and which can be treated by simple immobilization. Reduction may be achieved with a closed, percutaneous, intramedullary, or open technique ${ }^{(10,11)}$.

Nondisplaced or minimally displaced fractures ( $<30$ degrees of angulation, minimal translation) may be managed by simple immobilization of the elbow in 
a sling, posterior splint, or above-elbow cast for 1 to 2 weeks ${ }^{(12)}$.

In type II (30 to 60 degrees of angulation) and type III (>60 degrees of angulation) radial neck fractures, the attempt of closed reduction under conscious sedation or general anesthesia should be done. If the angulation could not be achieved to be less than 30 degrees, usually attempt of percutaneous or intramedullary reduction is the next step. Several authors have described using a $\mathrm{K}$-wire to joystick the proximal fragment into position percutaneously $(13,14,15,16)$.

Salter-Harris types III and IV injuries, as well as fractures that remain significantly angled after attempts at closed reduction and minimally invasive techniques, require open reduction.

Olecranon fracture complications are infrequent. Stiffness is the most common complication following an olecranon fracture. Fortunately, this is an unusual finding. Olecranon fractures have been associated with irreducible fractures, loss of reduction, delayed union and nonunion, peripheral nerve damage, and compartment syndrome ${ }^{(17,18)}$.

\section{CONCLUSION}

We achieved a close reduction of the radial neck and open reduction for the olecranon fractures. The patient tolerated the surgery well and she did many physiotherapy sessions to get full flexion, extension, and pronosupination, at 12 weeks follow up she achieved complete healing of olecranon and radial neck fracture (figure 4).

\section{ACKNOWLEDGMENT}

I would like to thank the patient for his cooperation and for his approval for making this case report.

Conflicts of interest: There are no conflicts of interest.

\section{REFERENCES}

1. Landin L, Danielsson L (1986): Elbow fractures in children. An epidemiological analysis of 589 cases. Acta Orthop Scand., 57:309.
2. Lindham S, Hugosson C (1979): The significance of associated lesions including dislocation in fractures of the neck of the radius in children. Acta Orthop Scand., 50:79.

3. Chambers H, Wilkins K (1996): Fractures of the olecranon. In Rockwood CA Jr, Wilkins K, Beaty J, editors: Fractures in children, ed 3, Philadelphia, Lippincott-Raven., p 613.

4. Fabry J, De Smet L, Fabry G (2000): Consequences of a fracture through a minimally ossified apophysis of the olecranon. J Pediatr Orthop B., 9:212.

5. Gicquel P, De Billy B, Karger C et al. (2001): Olecranon fractures in 26 children with a mean follow-up of 59 months. J Pediatr Orthop., 21:141.

6. Caterini R, Farsetti P, D'Arrigo C et al. (2002): Fractures of the olecranon in children. Long-term follow-up of 39 cases. J Pediatr Orthop B., 11:320.

7. Karlsson M, Hasserius R, Karlsson C et al. (2002): Fractures of the olecranon: a 15- to 25-year follow-up of 73 patients. Clin Orthop Relat Res., 403:205.

8. Zionts L, Moon C (2002): Olecranon apophysis fractures in children with osteogenesis imperfecta revisited. J Pediatr Orthop., 22:745.

9. Hope P, Williamson D, Coates C et al. (1991): Biodegradable pin fixation of elbow fractures in children. A randomized trial. J Bone Joint Surg Br., 73:965.

10. Goldenberg $R$ (1945): Closed manipulation for the resolution of fracture of the neck of the radius in children. $\mathbf{J}$ Bone Joint Surg., 27:267.

11. Murawski E, Stachow J (1977): Conservative reduction of radial bone neck fractures in children. Pol Przegl Chir., 49:117.

12. Sessa S, Lascombes P, Prevot J et al. (1996): Fractures of the radial head and associated elbow injuries in children. $\mathbf{J}$ Pediatr Orthop B., 5:200.

13. Bernstein S, McKeever P, Bernstein L (1993): Percutaneous reduction of displaced radial neck fractures in children. J Pediatr Orthop., 13:85.

14. Dormans J, Rang M (1990): Fractures of the olecranon and radial neck in children. Orthop Clin North Am., 21:257.

15. Radomisli T, Rosen A (1998): Controversies regarding radial neck fractures in children. Clin Orthop Relat Res., 353:30.

16. Rodriguez M (1994): Percutaneous reduction of displaced radial neck fractures in children. J Trauma, 37:812.

17. Matthews J (1980): Fractures of the olecranon in children. Injury, 12:207.

18. Zimmerman H (1980): Fractures of the elbow. In Weber BG, Brunner C, Freuler F, editors: Treatment of fractures in children and adolescents. New York, Springer-Verlag., p 158. 